\title{
RADIO SOURCES IN CLUSTERS OF GALAXIES
}

\author{
F.N. OWEN \\ NRAO \\ Socorro, NM, USA
}

\section{Introduction}

Since the Albuquerque IAU meeting, most of the advances in the understanding of radio sources in clusters has come from combining radio observations with $\mathrm{x}$-ray and optical data. The $\mathrm{x}$-ray images from Einstein and ROSAT have been particularly important because they have allowed us to see the external medium with which the radio sources co-exist and interact. I will cover three examples of such work in this review.

\section{Properties of Radio Sources in and out of Rich Clusters}

Much of the work reported in this section confirms the results of the studies of the Bologna group reported by Fanti (1984) but with much larger samples. Two recent Phd theses by Unewisse (1993) and Ledlow (1994) have given us large samples, in the southern and northern hemispheres respectively, with which to study the properties in rich clusters.

One important question is how do cluster properties affect the statistical properties of radio galaxies ? Both Ledlow's and Unewisse's results show that radio galaxy densities are very high in the centers of rich clusters, more peaked than the galaxy distribution. Ledlow's results (Ledlow, 1994; Ledlow \& Owen 1995, 1996) allow us to characterize the properties of cluster radio galaxies both in the radio and optical. When one considers the bivariate luminosity function, the observed radial distribution seems to be consistent with the clustering of the giant ellipticals about the cluster center.

His results also show that the probability of a galaxy being a radio source does not depend on richness, Bautz-Morgan class, or Rood-Sastry class. Furthermore, the bi-variate, fractional luminosity function of radio sources in Abell clusters are consistent in shape and amplitude with the luminosity function of all radio galaxies. Thus, once we take into account 
the optical luminosity of the galaxy, with the current evidence there is no apparent difference between galaxies in Abell clusters (the usual standard) and those in the "field". However, the most massive galaxies tend to live in clusters so some extreme phenomena tend to be found in rich clusters.

How could this be ? Models of extended radio emission rely on an external medium to provide the pressure which keeps the sources together. The radio luminosity depends on the magnetic field strength which in turn should depend on the external pressure. Thus the external medium should be critical to the size, luminosity and evolution of a radio source.

One possibility is that all radio galaxies live in some sort of a cluster and we just have missed them. In particular, Abell did not include them in his catalog. In order to test this idea we have used the ROSAT All Sky Survey (RASS) to look for evidence of extended $\mathrm{x}$-ray emission around a sample of all nearby $(z<0.05) 25$ radio galaxies, not in Abell clusters, chosen from the bright source list of Wall and Peacock (1985) or from the $3 \mathrm{CR}$. In 22 out of 25 cases we detected an extended $\mathrm{x}$-ray source greater or on-the-order of the size of the radio galaxy. The x-ray luminosity of the median source in this sample was four times weaker than than one finds for the median of Abell richness class 0, also using the RASS. Since we find that the median $\mathrm{x}$-ray luminosity for Abell clusters goes like the square of the number of galaxies Abell counted, then the x-ray luminosities and extents are consistent with cluster richness about half of what is typical for richness class 0 , or fifty percent less rich than the lower cutoff of Abell's definition.

Thus these results suggest that all radio galaxies, especially the FR I objects found which dominate our sample, live in some sort of cluster. It is just Abell's definition which has been confusing us. This makes it easier to understand how the luminosity functions and other properties could be so similar. This subject needs more study but these initial results are encouraging.

\section{Radio and X-ray Emission from Central Cluster Radio Galax- ies}

However, the very largest galaxies still are found at the centers of rich clusters. These galaxies, often extending hundreds of kiloparsecs in optical light are often to distinguish from the cluster potential and may not be physically distinct from it.

\subsection{CENTRAL GALAXIES IN COOLING FLOWS}

One class of such objects are galaxies at the centers of the class of clusters called "cooling flows". In these clusters there is a strong peak in the x-rays 
on the central galaxy which is caused by a decrease in temperature and a corresponding increase in density to preserve quasi-hydrostatic equilibrium. This structure can be simply interpreted as a slow radial inflow which compensates for the radiative energy losses in the cooling process.

However, at the centers of many strong cooling flows are radio sources, sometimes very powerful like Cygnus $\mathrm{A}$ and Hydra $\mathrm{A}$ and sometimes less luminous, very peculiar distorted sources. In the cases of Cygnus A (Harris, Carilli \& Perley 1994) and Perseus A (Böhringer et al 1993) comparison of the $\mathrm{x}$-ray and radio images show evidence for clear interaction of the radio emitting plasma with the hot external medium. In both of these cases it appears that the radio plasma has pushed the hot gas out of its path suggesting the radio jet is energetically important in the center of the cooling flow. To these two we add Abell 2199 (Eilek, Owen, and Zhou 1996). This source has for a long time been one of the most peculiar (see, for example Ge \& Owen 1993). The new ROSAT HRI image shows that the filamentary radio structure is wrapped around the base of a "unipolar" $\mathrm{x}$ ray structure suggesting a strong interaction, although it is not clear which medium is dominant.

These examples are starting to make a case that the centers of "cooling flow" clusters are not simple, passive, radial flows but must have a complex velocity field. Furthermore, the nuclear outflow in the form of a radio jet seems to be at least at times energetically important. Since the lifetime of the radio emission is thought to be much shorter than the cooling flow, it seems quite possible that this energy input may be of critical importance in causing the phenomena associated with cooling flows.

\subsection{WIDE-ANGLE-TAIL RADIO GALAXIES}

Another example of the peculiar radio sources associated with dominate central cluster radio galaxies are the Wide-Angle-Tail Sources (WATS). 3C 465 is the prototype. These sources consist of twin jets (or plumes if you prefer) coming from the center of the galaxies and then bending by an angle typically less than 90 degrees. They resemble the tailed sources attached to smaller cluster galaxies, like NGC 1265 , which are understood currently in terms of bending due to the motion of the galaxies in the cluster. However, since WATS are attached to central cluster galaxies which should be at rest, or nearly at rest with respect to the cluster, these bent sources have been harder to understand. See Eilek et al (1984) for a discussion of possible models.

However, recently ROSAT observations of the $\mathrm{x}$-ray gas associated with these systems have revealed that the $\mathrm{x}$-ray emission on the scale of the radio sources is almost always asymmetric and aligned with the direction 
toward which the tails are bending (Gomez et al, 1996). These results have been combined with the simulations of merging clusters first performed by Evrard (1990) and extended by others, e.g Schindler \& Müller (1993), Roettiger, Burns \& Loken (1993), to produce an explanation. In these simulations, which combine n-body and hydrodynamic codes, the changing gravitational potential produces large scale flows of hot gas in the cluster as well as complex $x$-ray structures like those often seen in WAT clusters. These flows appear to provide a wind flowing past the central galaxies which bend the WATS, although a detailed model of the interaction still needs to be constructed.

Thus in both the cooling flows and the WAT clusters, evidence now exists for strong interactions between the radio source and the hot cluster gas which are important for our understanding of the the total system.

\section{Radio Galaxies and the Butcher-Oemler Effect}

The cluster-cluster mergers simulations discussed in the previous section were motivated by cosmological arguments which suggest that such activity should be an ongoing part of the evolution of large scale structure in the universe. Both the common nature of substructure in nearby clusters and more general cosmological arguments suggest that galaxy clustering increases with time. Another possible outcome of this process is changing galaxy evolution with cosmological epoch. This could be due to changes with epoch in the cluster intergalactic medium and/or the probability of galaxy-galaxy mergers.

One possible piece of evidence for such processes affecting galaxy evolution is the systematic blueing of galaxy populations with epoch reported by Butcher and Oemler (1978), that is the Butcher-Oelmer (BO) effect. The blue population they report is supposedly dominated by galaxies that have undergone strong star formation in the last Gyr or so. On this timescale the galaxies have had time to move completely across the cluster and thus the location of the activity which caused the star formation is hard to pin down. Also once one gets away from the core of the cluster it is hard to pick out galaxies from the field which might be part of the activity. Thus if we want to look for star bursts and their cause actually in progress, we need some other signature of the process.

One good indicator should be the centimeter continuum emission at luminosities below $10^{23} \mathrm{~W} \mathrm{~Hz}^{-1}$ (at $20 \mathrm{~cm}, H_{0}=75 \mathrm{~km} \mathrm{~s}^{-1} \mathrm{Mpc}^{-1}$ ) where star formation dominates the galaxy luminosity function. The VLA can reach about $10^{22} \mathrm{~W} \mathrm{~Hz}^{-1}$ at $20 \mathrm{~cm}$ at a $z \sim 0.4$ in 24 hours integrations and thus can probe star formation to interesting redshifts. Furthermore the VLA primary beam is large enough, about 30 arcmin FWHM, at this wavelength 
to cover supercluster scales. The optical ID's with galaxies which would be bright enough to be star burst candidates found in such deep surveys are rare enough in a random field to pick out a good sample for further optical study from the background clutter, on supercluster scales.

To date we have studied two clusters with this technique: Abell 2125 (richness $=4, z=0.25$, blue fraction=0.19) and Abell 2645 (richness $=4$, $z=0.25$, blue fraction $=0.03$ ). The first results of this study are reported by Dwarakanath \& Owen (1996). In addition to the VLA data we have obtained optical spectra and colors at KPNO for a representative fraction of the objects. For Abell 2125, we have observed about $70 \%$ of the IDs brighter than about $R=19$. We find 20 radio galaxies so far in the cluster. For A2645, we have observed all but one of the IDs to a similar magnitude limit and find only four radio galaxies. The limiting radio luminosity is about $10^{22} \mathrm{~W} \mathrm{~Hz}^{-1}$. Thus there seems to be a large difference between the number of IDs in these two apparently similar clusters. Also the number of radio galaxies associated with Abell 2125 appears greatly to exceed any cluster known nearby.

However, it is not clear that all this radio activity in Abell 2125 is due to star formation. Only about half the IDs and less of the confirmed IDs have the very blue colors and/or the emission-line spectra to resemble nearby, strongly star forming, galaxies. The rest are red galaxies without obvious star formation signatures. They resemble FR I radio galaxies found nearby, although they make be slightly bluer than an uncontaminated old stellar population and we only have spectra covering wavelengths below about $6000 \AA$ in the galaxy rest frame. Thus on current evidence, the excess radio galaxy population seems to be made up of both star forming objects and objects one would guess are FR I-like.

A second interesting result is that the IDs in Abell 2125 are spread over a supercluster scale of at least $6 \mathrm{Mpc}$, where the VLA primary beam cuts off. The IDs occupy a band running from NE to SW exclusively. Furthermore, the most of the red galaxies are clustered nearer to the cluster center while there is a patch of blue radio galaxies centered about $2 \mathrm{Mpc} S W$ of the central galaxies. This pattern suggests to us that we could be looking at activity related to a cluster-cluster merger and that the Butcher-Oemler effect could be occurring on a much larger (supercluster) scale than the optical observations have so far indicated.

So far these results can only be looked at as suggestive. We need to see if the pattern repeats in more clusters and as a function of redshift. If it does it may indicate that the evolution of large scale structure not only stimulates star formation but also activity related to the nuclear sources and black holes which most of us believe are responsible for radio jets. Maybe the timescale for the jet activity to be stimulated is just longer 
than the star formation, which may begin before the galaxy-galaxy merger process is complete. Furthermore, if radio emission in galaxies is stimulated by large scale structure formation, it may be that this process is responsible for the strong evolution in the radio source luminosity function with epoch, not some local process isolated in the galaxy nucleus related to black hole formation.

\section{Conclusions}

1) Almost all radio galaxies seem to live in some sort of cluster-like environment.

2) The distorted structures of central cluster radio galaxies are due to their complex interaction with the thermal x-ray gas.

3) The evolution of radio source populations may be due to the evolution of the clustering environment with epoch.

\section{References}

Böhringer, H., Voges, W., Fabian, A.C., Edge, A.C., \& Neumann, D.M. (1993) MNRAS, 264, L25

Butcher, H.R. \& Oemler, A. (1978) ApJ, 219, 18

Dwarakanath, K.S. \& Owen, F.N. (1996) Butcher-Oemler Effect and Radio Continuum in Westerbork Synthesis Radio Telescope 25th Anniversary Workshop, Cold Gas at High Redshift in press

Eilek, J.A., Burns, J.O., O'Dea, C.P., \& Owen, F.N. (1984) ApJ, 278, 37

Eilek, J.A., Owen, F.N., \& Zhou, F. (1996) in preparation

Evrard, A.E. (1990) $A p J, 363,349$.

Fanti, R. (1984) Non Thermal Radio Sources in Clusters of Galaxies in Clusters and Groups of Galaxies, Reidel, Dordrecht p. 185

Ge,J-P \& Owen, F.N. (1993) $A J, 105,778$.

Gomez, P.L., Pinkney, J., Burns, J.O., Wang, Q., \& Owen, F.N. (1996), ApJ, submitted

Harris,D.E., Carilli, C.L., \& Perley, R.A. (1994) Nature, 367, 713

Ledlow, M.J. (1994) An Optical/Radio Study of Radio Galaxies In Abell Rich Clusters Phd thesis University of New Mexico

Ledlow, M.J. \& Owen, F.N. (1995) $A J, 109,853$

Ledlow, M.J. \& Owen, F.N. (1996), $A J$, submitted

Roettiger, K., Burns, J.O. \& Loken, C. (1993) $A p J, 407$, L53

Schindler, S. \& Müller, E. (1993) $A \& A, 272,137$

Unewisse, A.M. (1993) Radio Emission from Southern Clusters of Galaxies Phd thesis University of Sydney

Wall, J.V. \& Peacock, J.A. (1985) MNRAS, 216, 173 